\title{
Neumonía grave por infección con candida krusei: Reporte
} de caso

\section{Severe pneumonia due to infection with candida krusei: Case report}

\section{Autores:}

Ramiro Gutiérrez Vásquez. ${ }^{1}$, Alberto Ortega Rosales. ${ }^{2}$, Nelson Delgado Torres. ${ }^{3}$, Evelyn Zapata Tapia. ${ }^{4}$, Ricardo Calva Sánchez ${ }^{5}$.

\section{Recibido: 28-04-2019/ Revisado: 25-04-2019 /Aceptado: 24-06-2019/ Publicado: 05-07-2019}

\begin{abstract}
:
DOI: https://doi.org/10.33262/cienciadigital.v3i3.655

Pneumonia caused by Candida Krusei is a rare pathology, with a recent incidence increased. It is associated with a high mortality, and occurs especially in immunocompromised patients and geriatric patients. The diagnosis of this clinical entity is difficult due to nonspecific clinical presentation. We present a 90 -year-old patient who presented with acute respiratory symptoms, which did not evolve favorably after the administration of broad-spectrum antibiotics, but with complete remission of the clinical picture after the administration of voriconazole due to the clinical suspicion of pulmonary micosis. The definitive diagnosis of Candida Krusei pneumonia was established after confirming the growth of this germ in culture of bronchoalveolar lavage sample. The diagnosis of Candida pneumonia should be considered in patients with respiratory symptoms, especially in immunocompromised patients and geriatric patients. Timely treatment will avoid complications that compromise the patient's life.
\end{abstract}

Keywords: Pneumonia, Candida Pneumonia, Candida Krusei.

\section{Resumen:}

La neumonía por Candida Krusei es una patología rara, pero con reciente aumento de su incidencia. Se asocia a una alta mortalidad, y se presenta especialmente en pacientes inmunocomprometidos y pacientes geriátricos. El diagnóstico de esta entidad clínica es dificultoso debido a presentación clínica inespecífica. Presentamos

\footnotetext{
${ }^{1}$ Medihospital, Loja, Ecuador, ram,iroguti@hotmail.es

${ }^{2}$ Hospital General Marco Vinicio Iza, Lago Agrio, Ecuador, betoo.javi@gmail.com

${ }^{3}$ Hospital General Isidro Ayora, Loja, Ecuador, nelsondelgado81@ gmail.com

${ }^{4}$ Facultad de ciencias de la Salud UNL, Loja, Ecuador, evegabri16@gmail.com

${ }^{5}$ Hospital Básico Yantzaza, Loja, Ecuador, rjcalva8@gmail.com
} 
el caso de un paciente de 90 años de edad, que debutó con cuadro respiratorio agudo, que no evolucionó de forma favorable tras la administración de antibióticos de amplio espectro, pero con remisión completa del cuadro clínico luego de la administración de voriconazol debido a la sospecha clínica de micosis pulmonar, y que posteriormente se llegó al diagnóstico definitivo de neumonía por Candida Krusei al confirmarse el crecimiento de este germen en cultivo de muestra de lavado broncoalveolar. El diagnóstico de neumonías por Candida se debe considerar en pacientes con sintomatología respiratoria especialmente en inmunocomprometidos y pacientes geriátricos. El tratamiento oportuno permitirá evitar complicaciones que pongan en riesgo la vida del paciente.

Palabras clave: Neumonía, Neumonia Por Candida, Candida Krusei.

\section{Introducción}

La neumonía fúngica es un proceso infeccioso en los pulmones causado por uno o más hongos endémicos u oportunistas. La infección por hongos ocurre después de la inhalación de esporas, después de la inhalación de conidios, o por la reactivación de una infección latente (1). La diseminación hematógena ocurre de manera frecuente en un huésped inmunocomprometido. Los organismos fúngicos oportunistas (por ejemplo, especies de Candida, especies de Aspergillus) tienden a causar neumonía en pacientes con defectos congénitos o adquiridos en las defensas inmunitarias del huésped (2).

La cándida forma parte de la flora orofaringea normal y del tracto respiratorio superior. Sin embargo, la neumonía por Candida es una infección rara que se presenta con más frecuencia como parte de una micosis diseminada asociada con circunstancias clínicas predisponentes, como el uso prolongado de antibióticos, neoplasia hematológica o estados inmunosupresores graves (3) .

La neumonía debida a infección por Candida es una condición rara, con una incidencia aproximada del $0.4 \%$ (4) y por lo general asocia con un desenlace fatal, con una tasa de mortalidad de hasta el 70\% (5). La mayoría de los casos de neumonía por Candida son secundarios a la diseminación hematológica de los organismos de Candida desde un sitio distante, generalmente el tracto gastrointestinal o la piel (6)

En la literatura mundial se han reportado un número limitado de casos de neumonías por Candida. De forma particular, la infección fúngica por Candida krusei se presentan con menor frecuencia en comparación con otras especies de Candida. El diagnóstico de este tipo de micosis supone un enorme reto para el médico clínico (7), no solo por su rara aparición, sino también por su manifestación clínica inespecífica, siendo la tos, la fiebre y la taquipnea los síntomas más comunes. La expectoración de secreciones purulentas, hemoptisis y dolor torácico solo se presenta ocasionalmente (8).

La presentación radiológica tampoco es específica, y los signos radiográficos se pueden observar en muchas otras infecciones pulmonares oportunistas. Algunas neumonías fúngicas 
pueden manifestarse predominantemente como opacidades de vidrio esmerilado. También pueden estar presentes rasgos auxiliares, que incluyen linfadenopatía y derrames pleurales (9).

Realizar un diagnóstico convincente de la neumonía por Candida solo es realmente posible sobre la base de un examen microbiológico o histopatológico (10)

A continuación, presentamos un caso raro de neumonía por Candida krusei en un individuo adulto mayor con cuadro respiratorio agudo, con remisión completa de la sintomatología tras la administración de antifúngicos.

\section{Descripción del Caso}

Un paciente de sexo masculino de 90 años de edad, nacido en Ecuador, de ocupación agricultor, acude por presentar un inicio abrupto de tos no expectorante, sin causa aparente, que se acompaña de taquipnea y alza térmica; sin tratamiento para el cuadro. Dentro de los 6 días posteriores a la aparición de los síntomas, presentó exacerbación progresiva del cuadro clínico, además de expectoración amarillenta, disnea de medianos esfuerzos, malestar general y discreto edema en miembros inferiores. El historial médico del paciente indica antecedente de hipertensión arterial en los últimos 15 años, en tratamiento con Losartan, hipotiroidismo en tratamiento con levotiroxina, colocación bilateral de prótesis a nivel de rodilla hace 10 años y una colecistectomía hace 3 años.

$\mathrm{Al}$ ingreso, el paciente presentó fiebre (temperatura axilar de $37.8^{\circ} \mathrm{C}$ ) y ligera taquipnea $(24$ respiraciones/min, con una frecuencia cardíaca de 86 latidos/min, presión arterial de 96/62 mmHg y una saturación de oxígeno del $90 \%$ con $\mathrm{FiO} 2$ del 21\%. Al examen físico se evidenció disminución del murmullo vesicular, con presencia de estertores basales en ambos campos pulmonares. Corazón normofonético, sin ruidos sobreañadidos; a nivel de miembros inferiores se evidencia discreto edema. El examen neurogénico no mostró anomalías. En una RX de tórax se observa intersticiopatía bilateral.

Los exámenes de laboratorio realizados al ingreso revelaron los siguientes valores: hemoglobina, $11.2 \mathrm{mg} / \mathrm{dL}$; glóbulos blancos, $15.54 \times 10^{9} / 1(90.1 \%$ de neutrófilos, $13.7 \%$ de linfocitos y $8.7 \%$ de monocitos); y proteína C reactiva: $249.1 \mathrm{mg} / \mathrm{dl}$. El examen de gases arteriales demostró los siguientes resultados: pH 7.40; presión parcial de oxígeno, $62 \mathrm{mmHg}$; presión parcial de dióxido de carbono, $31.5 \mathrm{mmHg}$; $\mathrm{BE},-5 \mathrm{mmol} / \mathrm{l}$; nivel de bicarbonato, $19.7 \mathrm{mmol} / \mathrm{l}$. El análisis bioquímico de la sangre mostró los siguientes resultados: Aspartato aminotransferasa, $72.9 \mathrm{U} / \mathrm{l}$; potasio en sangre, $4.7 \mathrm{mmol} / \mathrm{l}$; Sodio en sangre, 138 mmol/l; creatinina, $1.62 \mathrm{mg} / \mathrm{dL}$; úrea, $58.2 \mathrm{mg} / \mathrm{dL}$. Serología para VIH y VDRL fue negativa. Paciente fue ingresado con diagnóstico de neumonía y se inicia antibioticoterapia con ceftriaxona 2 gramos intravenoso cada día, claritromicina $500 \mathrm{mg}$ intravenoso cada 12 horas y paracetamol 1 gramo intravenoso por razones necesarias. Se solicitó hemocultivos antes de administración de antibióticos. 
A los 3 días posteriores al ingreso se evidencia una pobre evolución clínica del paciente, continúa con picos febriles y requiere oxigenoterapia a 2 litros por minuto por bigotera nasal. En una tomografía axial computarizada simple de tórax se constata tenues opacidades en vidrio esmerilado a nivel basal bilateral [Fig. 1]. Se decide rotar de antibiótico a cefepime, cuya dosis se ajusta a función renal del paciente, y debido a la alta sospecha de una micosis pulmonar se prescribe voriconazol 200 miligramos intravenoso, dosis inicial, y luego $170 \mathrm{mg}$ cada 12 horas.

Fig. 1: TAC de tórax con tenues opacidades en vidrio esmerilado a nivel basal bilateral, asociado a bronquiectasias no retráctiles y engrosamiento pleural hacia los segmentos lateral y posterior del lóbulo inferior derecho

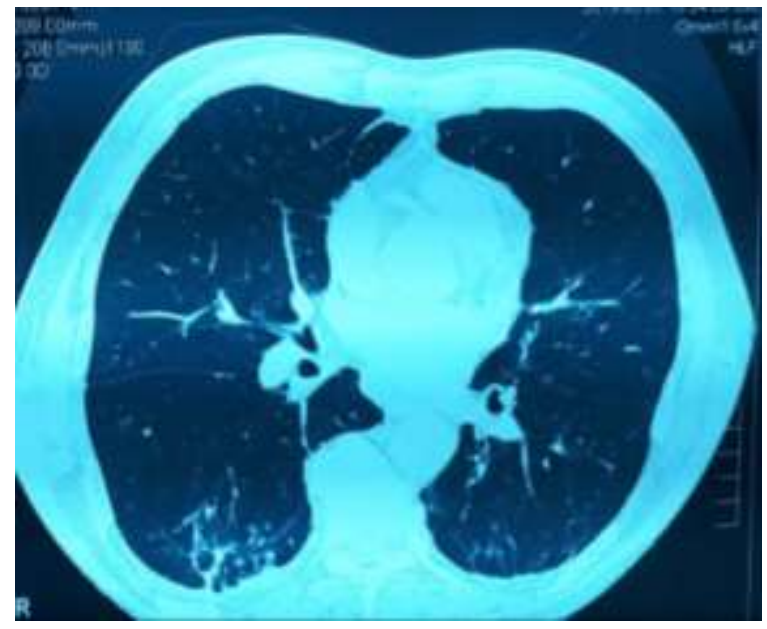

Se realiza fibrobroncoscopía diagnostica con cepillado y lavado bronquial, con envío de muestras para baciloscopía, cultivo de BAAR, $\mathrm{KOH}$, cultivo de hongos, cuantificación de galactomanano en lavado bronquial, cultivo de gérmenes comunes, estudio citológico. Se realizó examen de procalcitonina en sangre con un resultado de $0.77 \mathrm{ng} / \mathrm{ml}$.

A las 72 horas resultados de baciloscopías fueron negativos para Micobacterium Tuberculosis, $\mathrm{KOH}$ positivo, la detección de galactomanano en muestra de lavado bronquial fue negativa. Paciente luego de administración de antifúngico muestra una evolución favorable, remite cuadro febril Ventilatoriamente disminuye soporte de oxigeno por bigotera nasal, no se auscultan estertores crepitantes de mayor importancia en el examen físico realizado. En las últimas 24 horas no realiza alzas térmicas. Se mantiene plan terapeutico a base de cefepime y voriconazol ajustados a función renal.

Posteriormente en cultivo de muestra de lavado broncoalveolar se evidencia crecimiento de Candida krusei, [Fig. 2] por lo que se llega al diagnóstico definitivo de Neumonia por 
Candida krusei y debido al cuadro favorable del paciente se decide alta y administración de voriconazol por vía oral hasta 2 semanas posterior al alta. Paciente sin complicaciones posteriores.

Fig. 2: Cultivo de muestra de lavado broncoalveolar con crecimiento de Canida Krusei

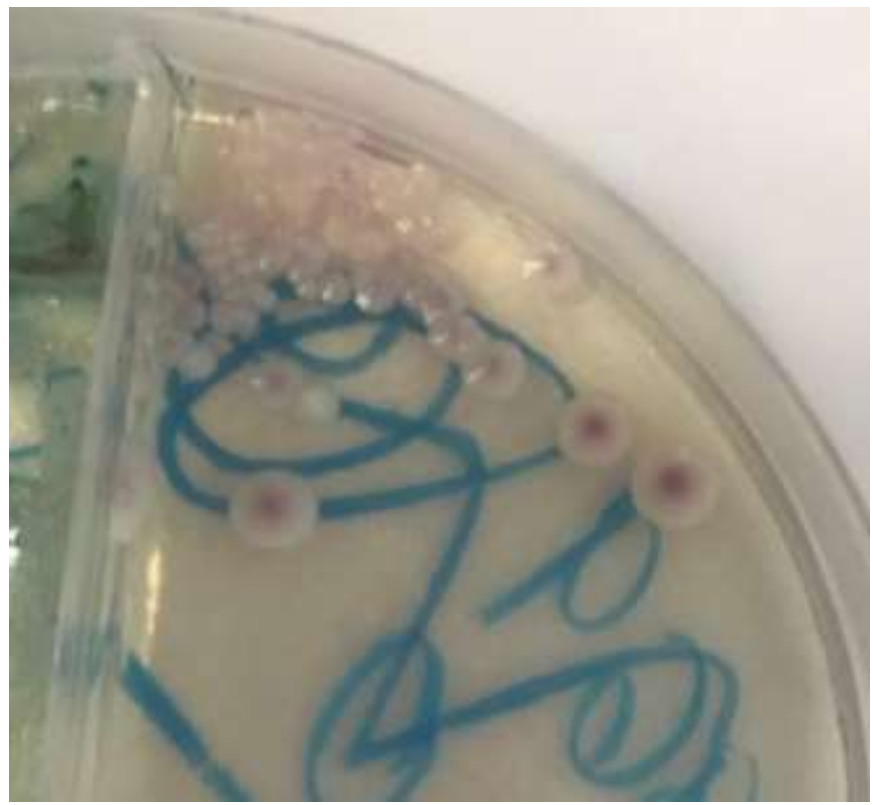

\section{Discusión}

C. krusei es un patógeno oportunista de la flora microbiana humana que puede causar infecciones pofundas especialmente en pacientes inmunocomprometidos, con alto riesgo de mortalidad (11). A pesar de que no constituye una de las especies aisladas con mayor frecuencia en pacientes infectados, la $C$. krusei es una causa de micosis invasiva con incidencia creciente (12).

Nuestro caso representa una ocurrencia singular de neumonía por C. krusei con una presentación poco frecuente. A diferencia de las presentaciones conocidas, incluidos los problemas relacionados con la broncoaspiración, la neumonía por infiltrado lobular o la diseminación hematológica, nuestro caso involucró una infección aislada del parénquima pulmonar sin evidencia de enfermedad sistémica, que es extremadamente rara y solo se describe en unos pocos informes previos (13).

Interesa, la edad del paciente y los antecedentes de hipertensión e hipotiroidismo que identificamos en este caso, que podrían guardar relación con el aumento del riesgo de infección (14). Sin embargo, esto no se puede confirmar claramente debido a la falta de 
diseminación e infección repetida. Por el contrario, la ausencia de infecciones repetidas o crónicas nos hace dudar si sus antecedentes patológicos y la edad del paciente son clínicamente relevantes. Alternativamente, la falta de inmunodeficiencia severa probablemente previno la diseminación y ayudó a contener una enfermedad a menudo fatal sin síntomas sistémicos aparentes.

El examen histopatológico de muestras de tejido obtenidas mediante procedimientos invasivos se considera el estándar de oro para el diagnóstico de neumonía por Candida. (15) Por ende, otro aspecto interesante de este caso fue la necesidad de un lavado broncoalveloar para asegurar un diagnóstico definitivo en base al cultivo de la muestra del lavado. Con un caso como el nuestro, nos preguntamos cómo se podría haber hecho un diagnóstico efectivo de otra manera.

Dado que el crecimiento de Candida en el esputo casi siempre se considera contaminación, los cultivos de esputo no son especialmente útiles. Asimismo, los modelos predictivos que diferencian la infección de la contaminación también son muy insensibles y no específicos. Por lo tanto, actualmente existe una necesidad clara de desarrollar un modelo clínico que permita la identificación y el tratamiento precisos y confiables de las infecciones por Candida sin la necesidad de realizar procedimientos invasivos.

Las infecciones invasivas por hongos y similares a los hongos se pueden sugerir en base a hallazgos radiográficos. Estas infecciones tienen un amplio espectro de características fenotípicas, que van desde nódulos hasta consolidaciones lobares e invasión de la pared torácica. Puede haber características de una bronconeumonía centrada en la vía aérea con nódulos asociados de árbol en brote. Algunas neumonías fúngicas pueden manifestarse predominantemente como opacidades de vidrio esmerilado.

También pueden estar presentes rasgos auxiliares, que incluyen linfadenopatía y derrames pleurales (9). La naturaleza manifiesta de muchos de los hallazgos radica en el desafío de distinguir los patrones característicos asociados con organismos particulares. Hay una superposición sustancial en los hallazgos, y puede tomar de días a semanas para que los cultivos den resultados. Sin embargo, el radiólogo puede agregar valor clínico al sugerir infecciones causadas por ciertos organismos sobre la base de características distintivas y, a su vez, facilitar la terapia dirigida.

En contraste, en el presente caso, en la TAC de tórax se evidenció un patrón en vidrio esmerilado, asociado a bronquiectasias no retráctiles y engrosamiento pleural hacia los segmentos lateral y posterior del lóbulo inferior derecho.

Finalmente el tratamiento antifúngico se instauró de manera oportuna en base al contexto clínico del paciente, permitiendo la remisión completa de la sintomatología, sin secuelas graves, por lo que consideramos que este estudio, en cierta medida, proporcionaría orientación o algunas experiencias al médico para diagnosticar una neumonía por Candida krusei en la práctica médica diaria. 


\section{Conclusiones:}

- La incidencia y la conciencia de las infecciones invasivas por hongos y similares a los hongos continúan aumentando a medida que la población de personas inmunocomprometidas crece y los tratamientos médicos mejoran. Estos organismos a menudo causan infecciones más indolentes en individuos inmunocompetentes, pero tienen una propensión a diseminarse rápidamente y pueden convertirse rápidamente en mortales en huéspedes inmunodeprimidos.

- La neumonía por Candida krusei se debe considerar en el diagnóstico diferencial de pacientes con sintomatología respiratoria, especialmente en aquellos con compromiso del sistema inmunológico, o pacientes añosos, cuyo diagnóstico definitivo se realiza en base al examen microbiológico. El tratamiento debe iniciarse de inmediato para mejorar la perfusión tisular y evitar posibles complicaciones que pongan en riesgo la vida del paciente.

- Algunas infecciones fúngicas y de tipo fúngico tienen apariencias de imagen específicas, mientras que otras se manifiestan con hallazgos más inespecíficos. Por lo tanto, es imperativo que los médicos tengan un umbral bajo para solicitar imágenes y que los radiólogos tengan un umbral bajo para sospechar una causa fúngica de la enfermedad en pacientes con alto riesgo de desarrollar estas infecciones.

\section{Referencias Bibliografía:}

1. Restrepo M, Faverio P, Anzueto A. Long-term prognosis in community-acquired pneumonia. Curr Opin Infect Dis. 2013; 26(2): p. 151-8.

2. Limper A. he changing spectrum of fungal infections in pulmonary and critical care practice: clinical approach to diagnosis. Proc Am Thorac Soc. 2010; 7(3): p. 163-8.

3. Shweihat Y, Perry J, Shah D. Isolated Candida infection of the lung. Respir Med Case Rep. 2015; 16: p. 18-19.

4. Masur H, Rosen P, Armstrong D. Pulmonary disease caused by Candida species. Am J Med. 1977; 6(3): p. 914-25.

5. Haron E, Vartivarian S, Anaissie E, Dekmezian R, Bodey G. Primary Candida pneumonia. Experience at a large cancer center and review of the literature. Medicine (Baltimore). 1993; 72(3): p. 137-42.

6. Pasqualotto A. Candida and the paediatric lung. Paediatr Respir Rev. 2009; 10(4): p. 186-191.

7. Meersseman W, Lagrou K, Spriet I, Maertens J, Verbeken E, Peetermans W, et al. Significance of the isolation of Candida species from airway samples in critically ill patients: a prospective, autopsy study. Intensive Care Med. 2009; 35(9): p. 1526-31.

8. Gogia P. Pulmonary fungal infections. Current Medicine Research and Practice. 2015; 5: p. 221-227. 
9. Connolly J, McAdams H, Erasmus J, Rosado-de-Christenson M. Opportunistic fungal pneumonia. J Thorac Imaging. 1999; 14(1): p. 51-62.

10. Schnabel R, Linssen C, Guion N, van Mook W, Bergmans D. Candida Pneumonia in Intensive Care Unit? Open Forum Infect Dis. 2014; 1(1): p. ofu026.

11. Galbán B, Mariscal F. [Epidemiology of candidemia in ICU]. Rev Iberoam Micol. 2006; 23(1): p. 12-5.

12. Fidan I, Yesilyurt E, Kalkanci A, Aslan S, Sahin N, Ogan M, et al. Immunomodulatory effects of voriconazole and caspofungin on human peripheral blood mononuclear cells stimulated by Candida albicans and Candida krusei. Am J Med Sci. 2014; 348(3): p. 219-23.

13. Knox K, Meinke L. Role of bronchoalveolar lavage diagnostics in fungal infections. Clin Chest Med. 2009; 30: p. 355-365.

14. el-Ebiary M, Torres A, Fàbregas N, de la Bellacasa J, González J, Ramirez J. Significance of the isolation of Candida species from respiratory samples in critically ill, non-neutropenic patients. An immediate postmortem histologic study. Am J Respir Crit Care Med. 1997; 156: p. 583-590.

15. Vélez L, Correa L, Maya M, Mejía P, Ortega j, Bedoya V, et al. Diagnostic accuracy of bronchoalveolar lavage samples in immunosuppressed patients with suspected pneumonia: analysis of a protocol. Respir Med. 2007; 101(10): p. 2160-7

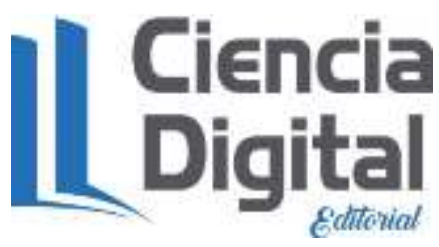


PARA CITAR EL ARTÍCULO INDEXADO.

Gutiérrez Vásquez, R., Ortega Rosales, A., Delgado Torres, N., Zapata Tapia, E., \& Calva Sánchez, R. (2019). Neumonía grave por infección con candida krusei: Reporte de caso. Ciencia Digital, 3(3), 422-430. https://doi.org/10.33262/cienciadigital.v3i3.655

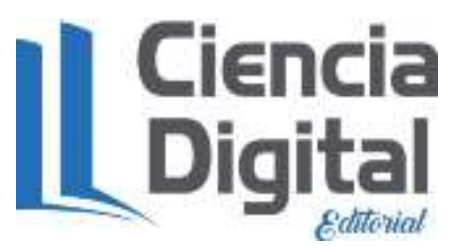

El artículo que se publica es de exclusiva responsabilidad de los autores y no necesariamente reflejan el pensamiento de la Revista Ciencia Digital.

El artículo queda en propiedad de la revista y, por tanto, su publicación parcial y/o total en otro medio tiene que ser autorizado por el director de la Revista Ciencia Digital.
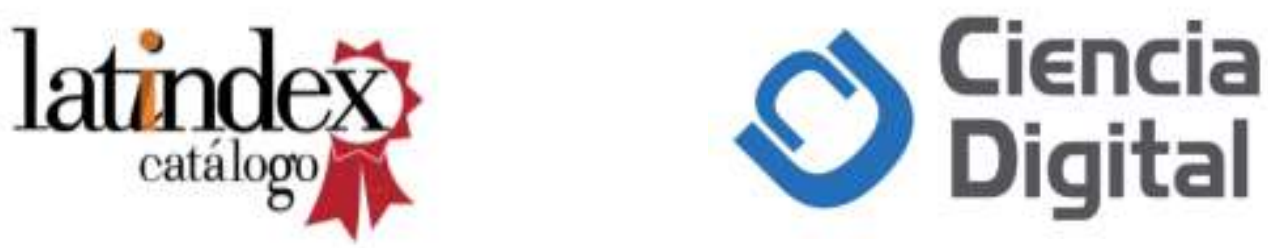